\title{
Impacto da Adoção de Mecanismos de Governança de Tecnologia de Informação (TI) no desempenho da Gestão da Tl: uma análise basea- da na percepção dos executivos
}

\author{
Guilherme Lerch Lunardi ${ }^{1}$ \\ João Luiz Becker ${ }^{2}$ \\ Antonio Carlos Gastaud Maçada ${ }^{3}$
}

\section{Resumo}

Recentemente surge na agenda dos executivos o termo governança de TI, aparecendo como uma tentativa de justificar os investimentos realizados em TI. Embora alguns estudos tenham levantado indícios de que empresas que possuem bons modelos de governança de TI apresentam resultados financeiros superiores aos de seus concorrentes, a questão sobre como implementar tais tecnologias na prática tem intrigado tanto executivos quanto acadêmicos. Assim, este trabalho pretende avaliar o impacto percebido da adoção de diferentes mecanismos de governança de TI no desempenho da gestão da TI. O estudo, realizado com 83 gestores da área de TI, permitiu identificar os mecanismos de governança de TI mais utilizados pelas suas empresas, bem como aqueles que mais afetam a gestão da TI. Os resultados apontaram vários mecanismos de governança de TI, apresentando correlação positiva significativa com diferentes áreas-foco da governança, destacando-se o Planejamento Estratégico de TI, os Comitês de projetos específicos de TI, a participação da área de TI na formulação da estratégia da empresa, as práticas de compliance e os comitês de TI como os principais, sugerindo que quanto mais efetivos forem esses mecanismos, melhor será o desempenho da gestão da TI.

Palavras-chave: Governança de TI. Gestão de TI. Mecanismos. Impacto. Desempenho.

\footnotetext{
${ }^{1}$ Doutor em Administração pela Escola de Administração da Universidade Federal do Rio Grande do Sul. Professor Adjunto do Instituto de Ciências Econômicas, Administrativas e Contábeis da Universidade Federal do Rio Grande. Endereço: Universidade Federal do Rio Grande (FURG), Instituto de Ciências Econômicas, administrativas e Contábeis/ICEAC. Endereço: Av. Itália, km 8, Bairro Carreiros. CEP: 96201-900. Rio Grande/RS-Brasil. E-mail: gllunardi@furg.br.

${ }^{2}$ Doutor em Management Science pela Anderson School of Managament da University of Califórnia at Los Angeles. Professor Titular do Departamento de Ciências Administrativas da Escola de Administração da Universidade Federal do Rio Grande do Sul. Universidade Federal do Rio Grande do Sul (UFRGS), Escola de Administração. Endereço: Rua Washington Luiz, 855. Centro. CEP: 90010-460. Porto Alegre/RS - Brasil. E-mail: jlbecker@ea.ufrgs.br.

${ }^{3}$ Doutor em Administração pela Escola de Administração da Universidade Federal do Rio Grande do Sul. Professor Adjunto do Departamento de Ciências Administrativas da Escola de Administração da UFRGS. Universidade Federal do Rio Grande do Sul (UFRGS), Escola de Administração. Endereço: Rua Washington Luiz, 855. Centro. CEP: 90010-460. Porto Alegre/RS-Brasil. E-mail: acgmacada@ea.ufrgs.br.

Artigo recebido em: 19/06/2010. Aceito em: 07/10/2010. Membro do Corpo Editorial Científico responsável pelo processo editorial: Thomas G. Brashear.
} 


\section{Introdução}

Sob uma perspectiva organizacional, a gestão da informação visa auxiliar a organização a atingir seus objetivos competitivos e estratégicos (DETLOR, 2010). A Tecnologia da Informação (TI), por sua vez, desempenha um papel crítico nas organizações, tornando-se parte integral das organizações modernas (NEVO; WADE, 2010). Deve ser ressaltado, entretanto, que a TI não é um fim em si mesma, representando tão somente o meio pelo qual as informações são armazenadas, acessadas, distribuídas e utilizadas. Assim, os executivos de TI devem preocupar-se com a gestão da informação, e não apenas com a gestão da TI. Essa nova orientação faz emergir o conceito de governança de TI.

Apontada em artigos e pesquisas ${ }^{4}$ - publicadas, na sua grande maioria, em revistas comerciais - como uma das principais prioridades das empresas brasileiras na atualidade, a governança de TI vem recebendo grande destaque entre acadêmicos e praticantes. O interesse dos executivos por essa temática tem se justificado pelo reflexo da mudança do papel (de predominantemente operacional para um papel mais estratégico e de apoio à decisão) e da relevância da TI nas organizações e, consequentemente, da necessidade de assegurar que ela seja adequadamente gerenciada. A governança de TI tem impacto direto sobre a gestão da TI, uma vez que é através dela que um conjunto de regras é elaborado e, a partir disso, tais regras são definidas, aplicadas e avaliadas para governar toda a função da TI na organização (VERHOEF, 2007).

A governança de TI, propriamente dita, envolve a aplicação de princípios de governança corporativa para dirigir e controlar a TI de forma estratégica, preocupando-se exclusivamente com dois assuntos-chave: o valor que a TI proporciona à organização e o controle e a diminuição dos riscos relacionados à TI (ITGI, 2003; PETERSON, 2004; HARDY, 2006). O primeiro assunto é direcionado pelo alinhamento estratégico entre os negócios e a tecnologia, enquanto o segundo é direcionado pela definição dos responsáveis na organização pelas decisões envolvendo os assuntos ligados à TI. Ambos precisam ser suportados por medidas e recursos adequados, para que os resultados desejados sejam alcançados (ITGI, 2003).

Medidas como a presença de comitês, a participação da área de TI na formulação da estratégia corporativa, os processos de elaboração e aprova-

\footnotetext{
4 "Governança de TI é prioridade para empresas brasileiras até 2008", Computerworld, 29/08/2007; e "Tendências 2007: que rumo toma a
} governança de TI", Computerworld, 26/12/2006. 
ção de orçamentos e projetos de TI, bem como o uso de modelos tradicionalmente encontrados na literatura especializada (como: COBIT, ITIL, ISO17799, SLA, PMI...) são apenas alguns mecanismos que procuram encorajar um comportamento consistente da organização, buscando alinhar os investimentos de TI com a missão, estratégia, valores e cultura organizacional (WEILL; ROSS, 2005). Em pesquisa realizada por Tarouco e Graeml (2009), com 51 grandes empresas brasileiras, pode- se que o conceito de governança de TI está ligado diretamente às iniciativas relacionadas ao aumento do controle e qualidade dos serviços prestados pela TI para a empresa.

Ainda que a governança de TI apareça como um meio de justificar e, principalmente, otimizar os investimentos realizados em tecnologia, a questão sobre como implementá-la na prática tem intrigado tanto executivos quanto acadêmicos (DE HAES; GREMBERGEN, 2009; WILLSON; POLLARD, 2009; BORGMAN; HEIER, 2010). A simples elaboração de um modelo não significa necessariamente que a governança de TI esteja funcionando na organização. Quando os processos de governança são mal projetados, eles acabam sendo contraproducentes. Meyer (2004), por exemplo, relata resultados de várias iniciativas de governança de TI malsucedidas (muitas vezes impondo processos complicados de fiscalização, prejudicando a organização e aumentando os custos administrativos).

Por outro lado, quando os processos de governança são bem planejados, cada aspecto da organização é coordenado: as pessoas, os equipamentos e os recursos financeiros trabalham em conjunto, em direção aos objetivos estratégicos da organização. Nesse sentido, estruturar e definir quais mecanismos de governança de TI devem ser implementados torna-se relevante para que as organizações possam gerenciar melhor os seus ativos de TI e, por conseguinte, obter maior retorno a partir dos investimentos que vêm realizando.

As pesquisas realizadas até o momento têm sido quase unânimes quanto a não existência de uma "melhor forma" de estruturar a TI, uma vez que a solução para qualquer organização é contingenciada por fatores como: tamanho da organização, ramo de atuação, localização geográfica, estrutura organizacional, estratégia e natureza da organização, dentre outros (BROWN; GRANT, 2005; BOWEN et al., 2007). Entretanto, a necessidade de aprofundamento de estudos sobre a adoção de mecanismos de governança e seus efeitos nas organizações é evidente, até mesmo porque além dos investimentos realizados na aquisição e manutenção da estrutura tecnológica da empresa, muitas organizações têm gasto elevadas quantias em consultorias, 
certificações, treinamentos e aquisições de softwares específicos para governarem a TI ("Governança movimenta R \$ 3,5 bilhões por ano", Computerworld, 17/10/2007). Peterson (2004) estabelece uma clara diferença entre a gestão da TI e a governança da TI. Enquanto a gestão da TI se atém ao fornecimento efetivo interno dos serviços e produtos de TI e no gerenciamento das suas operações, a governança de TI, por sua vez, é mais ampla, e se concentra na execução e na transformação da TI para atender às demandas presentes e futuras dos negócios (foco interno) e dos clientes desses negócios (foco externo). Acredita-se que a adoção de mecanismos de governança de TI acabe modificando o modo com que as organizações gerenciam e utilizam a sua tecnologia aplicada aos negócios de forma mais eficaz, refletindo, dessa forma, no desempenho global da organização (WEILL; ROSS, 2005).

Desse modo, pretende-se nesta pesquisa avaliar, sob a percepção dos executivos, o impacto da implementação de diferentes mecanismos de governança de TI no desempenho da gestão da TI. O estudo, realizado com 83 executivos, permitiu identificar os mecanismos de governança de TI que mais afetam a gestão da TI na percepção dos executivos, além de apresentar insights quanto à forma com que os mecanismos se correlacionam com diferentes aspectos da gestão da $\mathrm{TI}$. O artigo estrutura-se da seguinte forma: na Seção 2, apresenta-se uma visão geral da governança de TI e seus mecanismos, juntamente com o modelo de pesquisa proposto. Na Seção 3, descreve-se o método empregado no estudo; enquanto na Seção 4, são apresentados os principais resultados obtidos. O artigo se encerra na Seção 5, em que são apresentadas as conclusões e limitações do estudo.

\section{Governança de Tl e seus Mecanismos}

Segundo Peterson (2004), a governança de TI tem sido assunto de muito debate e especulação entre pesquisadores e praticantes há vários anos. Algumas questões e assuntos hoje ligados à governança de TI têm sido discutidos desde a introdução da TI nas organizações; entretanto, mesmo com mais de 30 anos de pesquisa empírica, ainda existem muitas brechas a serem preenchidas, especialmente por não existir um corpo teórico consistente e bem estabelecido sobre o tema.

A governança de TI e, mais especificamente, suas regras, são definidas com a finalidade de operacionalizar a função da TI na organização de forma 
mais eficiente e eficaz. A maioria dessas regras é resultado do senso comum, da padronização, da experiência e de "melhores práticas" aceitas pelo mercado (VERHOEF, 2007). Ainda assim, executivos de várias empresas acabam ignorando mesmo as regras mais básicas, como a elaboração de projetos de viabilidade de investimentos, ajustes periódicos do orçamento de acordo com as necessidades de negócio, uso de benchmarking ou, ainda, de métricas não financeiras, o que certamente influencia a forma como a TI é gerenciada e utilizada pela organização.

De modo geral, tem-se percebido uma grande insatisfação por parte dos executivos quanto à forma com que as decisões e os projetos de TI vêm sendo executados e justificados, o que tem levado pesquisadores, praticantes e até mesmo consultores de TI a proporem diferentes modelos, tendo por objetivo desenvolver e potencializar a governança de TI (McLANE, 2003; ALBERTIN, 2008). Segundo Van Grembergen, De Haes e Guldentops (2004), a governança de TI caracteriza-se por uma combinação de diferentes mecanismos associados à estrutura, processos e relacionamento (Quadro 1). Estruturas envolvem a existência de responsáveis pelas diferentes decisões de TI, além do uso de uma diversidade de comitês. Processos referem-se às técnicas e procedimentos ligados às estratégias de TI e seu monitoramento. Já os mecanismos de relacionamento incluem a participação da TI com as demais áreas de negócio, o diálogo estratégico, o aprendizado compartilhado e a comunicação apropriada. Lunardi e Dolci (2009) apresentam uma breve descrição dos principais mecanismos de governança de TI encontrados na literatura, indicando os mais difundidos entre as empresas brasileiras.

\begin{tabular}{l|l|l|}
\multicolumn{1}{|c|}{ Estruturas } & \multicolumn{1}{|c|}{ Processos } & \multicolumn{1}{|c|}{ Mecanismos de Relacionamento } \\
\hline - Papéis e responsabilidades & - Indicadores de desempenho & - Participação ativa de principais stakeholders \\
- Comitê de Estratégia de TI & de TI & - Colaboração entre principais stakeholders \\
- Comitê Diretivo de TI & - Planejamento Estratégico de & - Incentivos e recompensas \\
- Estrutura Organizacional & Sistemas de Informação & - Colocação de TI e de negócios \\
da TI & - COBIT & - Compreensão compartilhada dos \\
- CIO no Conselho de & - ITIL & objetivos de TI e de negócios \\
Administração & - Acordos de Nível de Serviço & - Resolução ativa de conflitos \\
- Comitê de projetos de TI & - Métodos de avaliação de & - Treinamento interfuncional entre TI e \\
- Escritório de projetos & retorno de investimento & negócios \\
& - Avaliação ex post & - Rotação de tarefas de TI e negócios \\
\hline
\end{tabular}

Quadro 1: Diferentes mecanismos de governança de TI

Fonte: Adaptado de Peterson (2004) 
Cada um desses mecanismos se destina a um ou mais objetivos da governança - seja diminuir riscos, gerar maior valor para a organização, ou até mesmo, garantir que os investimentos estejam alinhados à estratégia corporativa. A literatura de Sistemas de Informação (SI) destaca diferentes pontos - também chamados de áreas-foco - que devem ser observados pelas organizações ao governarem sua TI, sendo os mais frequentemente citados o alinhamento estratégico entre a TI e os negócios, o valor entregue pela TI à organização, o gerenciamento dos riscos relacionados à $\mathrm{TI}$, o gerenciamento de recursos de $\mathrm{TI}$, a mensuração do desempenho da TI e a responsabilidade pelas decisões de TI (também chamada de accountability) (VAN GREMBERGEN; DE HAES; GULDENTOPS, 2004; GWILLIM; DOVEY; WIEDER, 2005; ITGI, 2005; BROWN, 2006; HARDY, 2006; WEBB; POLLARD; RIDLEY, 2006; GELLINGS, 2007; ISACA, 2008; WILLSON; POLLARD, 2009; BRETERNITZ et al., 2009).

Acredita-se que as organizações que apresentam boa governança de TI são aquelas que possuem elevado desempenho nessas diferentes áreas-foco, obtendo, portanto, maior retorno sobre os investimentos realizados em TI do que as empresas que não conseguem governar sua TI de forma adequada. Ao avaliar cada uma dessas áreas, a organização obtém um retrato de quão bem vem gerenciando seus recursos tecnológicos da informação, além de identificar os efeitos da adoção de tais mecanismos na gestão da TI.

A partir da revisão de artigos e livros da área de SI, os quais destacam diversos pontos a serem observados pelas organizações ao governarem sua TI, propõe-se um modelo (Figura 1) capaz de avaliar seis diferentes fatores que promovem a efetividade da governança de TI em uma organização, quais sejam:

a) Alinhamento estratégico (AE): busca assegurar a integração entre o plano de TI e o de negócios, de modo a manter as soluções e estratégias de TI alinhadas às estratégias e aos negócios da empresa.

b) Valor da TI (VA): busca assegurar que a TI esteja atingindo seus benefícios prometidos, otimizando os custos dos investimentos.

c) Gerenciamento de risco (RIS): busca proteger os ativos de TI, recuperando informações em caso de desastres e mantendo a continuidade das operações dos serviços de TI. 
d) Recursos de TI (REC): busca otimizar a infraestrutura e o conhecimento de TI da empresa. Trata também dos recursos críticos de TI (aplicativos, informação e pessoal).

e) Medidas de Performance (MP): busca acompanhar e monitorar a implementação da estratégia e dos projetos de TI, bem como dos recursos de TI utilizados e demais serviços relacionados à TI.

f) Accountability (AC): tem por objetivo definir os papéis e responsabilidades das partes envolvidas nas decisões de TI, além de assegurar claramente a sua compreensão pela organização.

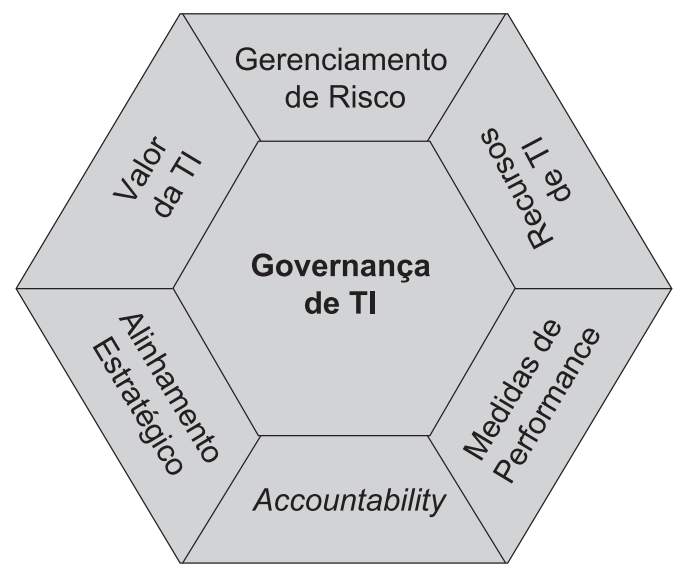

Figura 1: Modelo de efetividade da Governança de TI Fonte: Elaborada pelos autores

Independentemente de estarem engajadas formalmente no processo de implementação da governança de TI, as organizações, em geral, apresentam variados níveis de maturidade. Essa maturidade pode ser avaliada pela utilização que a organização faz dos diferentes mecanismos de governança de TI no seu dia a dia - algumas desenvolvem seu próprio modelo de governança, outras acabam implementando uma ou mais metodologias já consolidadas no mercado (como o COBIT e o ITIL); e ainda existem aquelas empresas cujo processo de governança ainda não é formalizado, utilizando, entretanto, alguns desses mecanismos, em maior ou menor quantidade.

À medida que a utilização desses mecanismos vai se tornando melhor compreendida e seu uso mais frequente, maior é o nível de maturidade da governança de TI, tendo como um dos seus principais benefícios entender 
melhor os investimentos realizados, bem como verificar se esses investimentos vêm atingindo os resultados esperados (VAN GREMBERGEN; DE HAES; GULDENTOPS, 2004). Esses mecanismos, por sua vez, não necessariamente precisam ser utilizados na sua totalidade ou da mesma forma pelas organizações. Uma série de características da própria empresa ou negócio de atuação pode exigir diferentes configurações, evidenciando a complexidade na determinação dos mecanismos mais indicados a serem adotados.

\section{Metodologia}

O estudo caracteriza-se como uma pesquisa survey, realizada com 81 empresas nacionais de grande porte, cujos respondentes $(n=83)$ são os responsáveis pela área de TI de suas respectivas empresas. A pesquisa iniciou-se no segundo semestre de 2007 (encerrando-se em fevereiro de 2008), envolvendo uma etapa exploratória, na qual elaborou-se o instrumento de coleta de dados, e outra quantitativa, englobando procedimentos de amostragem, coleta, validação e análise de dados. Os procedimentos metodológicos seguidos na pesquisa são descritos a seguir.

\subsection{Etapa Exploratória}

O instrumento de coleta de dados foi elaborado a partir de uma extensa revisão da literatura, levando em consideração essencialmente as seis dimensões propostas para avaliar a efetividade da governança de TI. Após a definição das dimensões, procedeu-se à conversão formal dos construtos definidos em variáveis mensuráveis. Foram pesquisados na literatura diferentes aspectos observados pelas organizações ao governarem sua TI, resultando na proposição de 35 variáveis candidatas.

As variáveis foram adaptadas em forma de questão e agrupadas conforme sua associação com os construtos propostos. De modo a realizar a validade de face dos itens identificados na literatura, empregou-se a técnica de classificação de cartões (do inglês q-sort) em forma de painel, junto a oito especialistas da área de SI (SEGARS; GROVER, 1998). O q-sort classificou quatro dos seis construtos com elevado percentual de convergência quanto à forma com que as questões haviam sido inicialmente classificadas 
(gerenciamento do risco $=100 \%$; valor da $\mathbf{T I}=100 \%$; medidas de performance $=83 \%$; e alinhamento estratégico $=80 \%$ ), pois os itens referentes à accountability (40\%) e aos recursos de TI (43\%) mostraramse menos coesos. Os enunciados que haviam apresentado baixa convergência entre os construtos foram submetidos a uma análise junto a outros dois especialistas que sugeriram pequenas modificações nas questões que acabaram não convergindo nas dimensões inicialmente propostas. Das 35 variáveis candidatas, uma foi eliminada, duas foram realocadas e outras seis tiveram seu enunciado modificado, porém mantiveram-se nos seus respectivos construtos.

Logo após, procedeu-se à estruturação e diagramação do questionário estruturado que foi dividido em três blocos, contendo: (1) Informações gerais: com nove questões de caracterização da empresa e do respondente; (2) Mecanismos de governança de TI: contendo uma lista de 24 mecanismos de governança de TI (identificados em mais de 100 anúncios publicados eletronicamente sob a forma de artigos, balanços contábeis, cases, entrevistas, mesas-redondas, notícias e sites institucionais), operacionalizados em uma escala de intensidade de cinco pontos (variando de "nenhum" a "muito grande") e identificando o impacto de cada mecanismo - quando presente - na gestão de TI da empresa; e (3) Gestão da TI: contendo 34 questões relacionadas às seis dimensões propostas, todas operacionalizadas em uma escala de cinco pontos (variando de "discordo totalmente" a "concordo totalmente"). O instrumento foi submetido a um pré-teste com três especialistas: sendo dois deles CIOs de duas grandes empresas nacionais (listadas na BOVESPA e que estavam implementando modelos formais de governança de TI) e o terceiro, consultor de uma empresa especializada na implementação de modelos e mecanismos de governança de TI.

\subsection{Etapa Quantitativa}

A partir do questionário previamente elaborado, ele foi novamente testado sob a forma de um estudo-piloto, sendo aplicado, desta vez, em um grupo maior de respondentes, de modo a identificar possíveis problemas de formatação ou compreensão das questões inseridas no questionário. $\mathrm{O}$ estudo-piloto foi realizado com membros do GUCIO-RS, que é um grupo de CIOs de grandes empresas do Rio Grande do Sul que se reúne periodicamente para discutir e trocar experiências sobre temas ligados à área de TI. Foram 
obtidas respostas de $22 \mathrm{CIOs,}$ as quais serviram de base para verificar a confiabilidade e a validade dos construtos propostos.

Foram seguidos os procedimentos estatísticos indicados por Churchill (1979), os quais sugerem a avaliação da confiabilidade do instrumento e dos construtos por meio do coeficiente Alfa de Cronbach, e a avaliação convergente dos itens pela análise de correlação item-total corrigida (CITC). Os coeficientes dos seis fatores analisados situaram-se entre 0,73 e 0,82, mostrando boa consistência interna do instrumento, especialmente pela natureza e estágio da pesquisa. Como o valor do alfa se manteve acima do limite sugerido pela literatura para estudos exploratórios $(0,60)$, optou-se pela não exclusão de itens nessa etapa inicial, permitindo o aproveitamento dos 22 questionários recebidos na análise final do estudo.

Após a realização do estudo-piloto, procedeu-se a sua aplicação e validação. Um e-mail foi enviado aos departamentos de Relações com Investidores de todas as empresas listadas na Bolsa de Valores de São Paulo, solicitando o seu encaminhamento aos responsáveis pela área de TI da empresa. $\mathrm{O}$ e-mail detalhava a pesquisa e solicitava ainda que a empresa participasse do estudo, respondendo a um questionário disponibilizado em forma eletrônica. No total, 61 questionários (de um total aproximado de 400) retornaram corretamente preenchidos. Aos que voltaram preenchidos, foram adicionados os 22 questionários utilizados no estudo-piloto, o que totalizou 83 casos. Vale lembrar que oito dos 22 questionários obtidos no estudo-piloto foram preenchidos por executivos de empresas listadas na BOVESPA, o que representa $83 \%(n=69)$ dos respondentes.

Após o término da coleta de dados, procedeu-se à validação do instrumento. Cabe destacar que a classificação de cartões e o estudo-piloto que foram realizados como forma de validar o conteúdo das escalas (que sem dúvida auxiliou na compreensão e identificação de pequenos problemas no instrumento), dada a sua natureza predominantemente subjetiva, não se caracterizam como medidas suficientes para validar os construtos propostos, exigindo a realização de outros testes estatísticos. Procedeu-se, inicialmente, à análise de fidedignidade das escalas, através da realização do alfa de Cronbach e da correlação item-total corrigido (CITC). Neste último procedimento, utilizam-se os coeficientes de correlação entre cada item e o escore corrigido (escore do grupo menos o escore do item) de seu grupo, indicando o grau de correlação entre eles. Esse procedimento eliminou seis itens do instrumento, sendo dois relativos à variável Gerenciamento de Recur- 
sos, dois pertencentes ao construto Valor da $\mathbf{T I}$, um relativo ao Gerenciamento de Risco e um relativo ao construto Accountability.

Subsequentemente, utilizou-se a Análise Fatorial Exploratória (AFE) nos blocos (Tabela 1), de modo a se observar a unidimensionalidade dos construtos. Segundo Koufteros (1999), poucos pesquisadores têm feito uso da AFE nos blocos, o que é lamentável, uma vez que o seu resultado é bastante informativo. A partir dessa análise, percebeu-se que os itens de cada construto, analisados conjuntamente, convergiam para um único fator - sugerindo serem todos unidimensionais.

Em seguida, foram submetidos os 28 itens restantes à análise fatorial exploratória entre blocos (Tabela 1), buscando verificar a validade discriminante do instrumento. Definiu-se como método de extração a análise de componentes principais (ACP), aplicando-se a rotação ortogonal Varimax para discriminar melhor a pertinência das variáveis aos componentes identificados. A formação dos fatores obedeceu a dois critérios: o grau de associação entre as variáveis, encontrado através das cargas fatoriais; e o seu grau de subjetividade. Ao final dessa análise, cinco itens foram excluídos por não apresentarem coerência conceitual ou apresentarem baixas correlações com os demais itens de seu grupo.

Uma última apreciação da fidedignidade do instrumento foi realizada após a execução da AFE entre blocos, não sugerindo a eliminação de outros itens do questionário. Em sua versão final, o instrumento manteve os seis construtos propostos, sendo constituídos por 23 itens. $\mathrm{O}$ coeficiente de fidedignidade (alfa de Cronbach) do instrumento apresentou valor 0,91, enquanto os coeficientes dos fatores situaram-se entre 0,73 e 0,85 , apontando boa consistência interna do instrumento (Tabela 1). 
Tabela 1: Análise Fatorial Exploratória

\begin{tabular}{|c|c|c|c|c|c|c|c|}
\hline \multirow{3}{*}{ Indicadores } & \multicolumn{7}{|c|}{ Cargas Fatoriais } \\
\hline & \multirow[t]{2}{*}{ Bloco } & \multicolumn{6}{|c|}{ Entre Blocos } \\
\hline & & F1 & F2 & F3 & F4 & F5 & F6 \\
\hline \multicolumn{8}{|l|}{ Medidas de Performance } \\
\hline Q27. Todos os projetos de TI são entregues no prazo & ,78 &, $\mathbf{8 1}$ &, 13 & 01 &,- 02 & ,20 & 17 \\
\hline $\begin{array}{l}\text { Q33. Os serviços e as soluções de TI são entregues sem } \\
\text { defeitos e problemas }\end{array}$ & 77 &, 70 & ,22 & ,20 &,- 05 & ,19 &,- 06 \\
\hline Q21. Os projetos de TI não ultrapassam o orçamento previsto &, 75 &, 68 & ,20 & ,09 &, 10 &,- 10 & 23 \\
\hline $\begin{array}{l}\text { Q12. Os serviços terceirizados de TI são constantemente } \\
\text { monitorados e ajustados quando necessário }\end{array}$ & ,73 &, 59 & ,09 & ,23 & ,41 &, 16 &, 10 \\
\hline Q17. Os projetos de TI têm atingido os benefícios esperados & ,71 &, 54 &,- 09 & ,51 & ,30 & ,20 &,- 01 \\
\hline \multicolumn{8}{|l|}{ Gerenciamento de Risco } \\
\hline $\begin{array}{l}\text { Q25. A infraestrutura de TI e as informações da empresa } \\
\text { estão bem protegidas e seguras }\end{array}$ & ,93 & ,22 &, 82 & ,11 & ,07 & ,28 &, 08 \\
\hline $\begin{array}{l}\text { Q30. A infraestrutura de TI assegura e mantém a integridade } \\
\text { das informações }\end{array}$ & ,91 & 25 &, 81 & 00 & 22 & ,22 &, 01 \\
\hline $\begin{array}{l}\text { Q13. Informações confidenciais são impedidas de serem } \\
\text { acessadas por pessoas não autorizadas }\end{array}$ &, 81 & ,08 &, 81 &, 17 & ,11 &,- 03 &, 01 \\
\hline \multicolumn{8}{|l|}{ Alinhamento Estratégico } \\
\hline $\begin{array}{l}\text { Q3. A TI está em harmonia com os objetivos e necessidades } \\
\text { organizacionais estabelecidos pela alta administração }\end{array}$ & ,89 & ,19 & ,12 & ,78 & ,16 &, 03 & ,27 \\
\hline $\begin{array}{l}\text { Q8. As soluções de TI implementadas pela organização estão } \\
\text { alinhadas aos negócios da empresa }\end{array}$ & 88 &, 15 & ,11 & ,75 & ,22 & ,34 & 13 \\
\hline $\begin{array}{l}\text { Q20. Os projetos de TI da empresa estão alinhados às } \\
\text { estratégias organizacionais }\end{array}$ & 84 & ,24 & ,22 & ,63 &, 15 & ,04 & ,41 \\
\hline \multicolumn{8}{|l|}{ Gerenciamento de Recursos } \\
\hline $\begin{array}{l}\text { Q11. As operações da organização são automatiza das de } \\
\text { forma bastante satisfatória }\end{array}$ & ,82 &,- 02 &, 18 & ,16 &, 77 &, 17 &, 04 \\
\hline $\begin{array}{l}\text { Q5. As habilidades computacionais dos usuários respondem à } \\
\text { estratégia de TI da organização }\end{array}$ & ,79 & ,06 &,- 01 & ,30 &, 72 &, 02 &, 17 \\
\hline $\begin{array}{l}\text { Q6. A infraestrutura e os serviços de TI podem resistir e se } \\
\text { recuperar de falhas decorrentes de erros, desastres e ataques } \\
\text { deliberados (como hackers e vírus) }\end{array}$ & ,76 &, 19 & ,40 &,- 13 &, 60 &, 17 & ,33 \\
\hline $\begin{array}{l}\text { Q26. Os recursos de TI (hardware, software, pessoal) são } \\
\text { adequados para suportar as aplicações de negócios }\end{array}$ & ,70 &, 44 &, 17 & ,17 &, 53 &, 19 & ,29 \\
\hline \multicolumn{8}{|l|}{ Valor da TI } \\
\hline Q7. A TI aumenta a eficiência operacional da empresa & ,76 &, 04 &, 09 &,- 03 & ,11 &, 78 & ,27 \\
\hline Q29. A TI mantém a empresa competitiva & 81 & ,30 & ,13 & ,18 & ,19 & ,73 &, 02 \\
\hline Q14. A TI auxilia a empresa a reduzir custos & ,77 &, 10 &, 48 & ,18 &,- 05 &, 65 &, 11 \\
\hline $\begin{array}{l}\text { Q31. A TI a umenta a qualidade dos produtos e serviços } \\
\text { produzidos pela empresa }\end{array}$ & ,76 & ,08 & 06 & ,36 & ,39 & ,58 &,- 16 \\
\hline \multicolumn{8}{|l|}{ Accountability } \\
\hline $\begin{array}{l}\text { Q10 Todos os projetos de TI têm pessoas responsáveis pelos } \\
\text { seus resultados e seu acompanhamento }\end{array}$ & 82 & 28 &,- 06 & 14 & ,21 & ,05 & ,77 \\
\hline Q15 O papel da TI na empresa é bem claro &, 82 & ,01 & ,09 &, 35 & ,13 & 25 & ,70 \\
\hline $\begin{array}{l}\text { Q18. Todas as decisões sobre a TI e seus investimentos são } \\
\text { tomadas de forma consistente (elaboração, seleção, } \\
\text { priorização e aprovação de projetos) }\end{array}$ & ,79 & 49 &, 14 & ,32 &, 11 &, 01 &, $\mathbf{5 0}$ \\
\hline Initial eigenvalue & & 7,98 & 2,12 & 1,72 & 1,35 & 1,16 & 1,06 \\
\hline \% variância explicada - rotated $(70,0 \%)$ & & 36,3 & 9,6 & 7,8 & 6,1 & 5,3 & 4,8 \\
\hline $\begin{array}{l}\text { Alfa de Cronbach }(0,91) \\
\text { KMO medida de adequação da amostra }(\mathrm{KMO}=0,82) \\
\text { Teste de Bartlet: qui-quadrado }=822,620\end{array}$ & & 0,80 & 0,85 & 0,84 & 0,77 & 0,76 & 0,73 \\
\hline
\end{tabular}

Fonte: Elaborada pelos autores 


\subsection{Caracterização da Amostra}

A amostra é composta por 83 executivos de TI de 81 empresas. Em apenas duas empresas, mais de um executivo de TI respondeu ao questionário; nas demais, somente um responsável preencheu o questionário em nome da sua empresa. As organizações estudadas estão presentes em 26 subsetores da Economia (Tabela 2), conforme classificação da BOVESPA. Predominaram as empresas dos setores bancário (11), energia elétrica (9), máquinas e equipamentos (7), químico (6), material rodoviário (5) e construção (5), que juntos representam quase metade das empresas estudadas.

Tabela 2: Classificação das empresas da amostra por subsetor da Economia

\begin{tabular}{|c|c|c|c|}
\hline Subsetor da Economia & $\mathrm{n}$ & $\%$ & $\%$ Acumulado \\
\hline Bancos & 11 & $13,6 \%$ & $13,6 \%$ \\
\hline Energia Elétrica & 9 & $11,1 \%$ & $24,7 \%$ \\
\hline Máquinas e Equipamentos & 7 & $8,6 \%$ & $33,3 \%$ \\
\hline Químicos & 6 & $7,4 \%$ & $40,7 \%$ \\
\hline Material Rodoviário & 5 & $6,2 \%$ & $46,9 \%$ \\
\hline Construção & 5 & $6,2 \%$ & $53,1 \%$ \\
\hline Tecnologia & 4 & $4,9 \%$ & $58,0 \%$ \\
\hline Alimentos & 4 & $4,9 \%$ & $63,0 \%$ \\
\hline Siderurgia e Metalúrgica & 4 & $4,9 \%$ & $67,9 \%$ \\
\hline Transporte & 3 & $3,7 \%$ & $71,6 \%$ \\
\hline Tecidos, Vestuário e Calçados & 2 & $2,5 \%$ & $74,1 \%$ \\
\hline Exploração de Rodovias & 2 & $2,5 \%$ & $76,5 \%$ \\
\hline Telefonia Móvel & 2 & $2,5 \%$ & $79,0 \%$ \\
\hline Telefonia Fixa & 2 & $2,5 \%$ & $81,5 \%$ \\
\hline Petróleo e Gás & 2 & $2,5 \%$ & $84,0 \%$ \\
\hline Uso pessoal e de Consumo & 2 & $2,5 \%$ & $86,4 \%$ \\
\hline Governo & 2 & $2,5 \%$ & $88,9 \%$ \\
\hline Eletrodomésticos & 1 & $1,2 \%$ & $90,1 \%$ \\
\hline Embalagens & 1 & $1,2 \%$ & $91,4 \%$ \\
\hline Saúde & 1 & $1,2 \%$ & $92,6 \%$ \\
\hline Seguros & 1 & $1,2 \%$ & $93,8 \%$ \\
\hline Mídia & 1 & $1,2 \%$ & $95,1 \%$ \\
\hline Comércio Varejista & 1 & $1,2 \%$ & $96,3 \%$ \\
\hline Sociedade de Crédito & 1 & $1,2 \%$ & $97,5 \%$ \\
\hline Papel e Celulose & 1 & $1,2 \%$ & $98,8 \%$ \\
\hline Holdings & 1 & $1,2 \%$ & $100,0 \%$ \\
\hline Total & 81 & $100,0 \%$ & $100,0 \%$ \\
\hline
\end{tabular}

Fonte: Elaborada pelos autores 
Com relação ao perfil dos respondentes, 43 (51,8\%) eram gerentes de TI, $18(21,7 \%)$ diretores de TI e os demais se distribuíam entre coordenadores, analistas, superintendentes, vice-presidentes, consultores e especialistas de TI. Observando o número de empresas que possuem modelos formais de governança de TI, percebeu-se um equilíbrio entre as empresas que afirmaram possuir um modelo formal $(45 ; 54,2 \%)$ e aquelas que afirmaram apenas possuir alguns dos mecanismos de governança de TI, sem possuírem, entretanto, um modelo específico $(38 ; 45,8 \%)$.

\section{Resultados e Discussão}

Esta seção apresenta os principais resultados que foram obtidos no estudo. Inicialmente, procede-se à descrição dos mecanismos de governança de TI apontados pelos executivos como os que mais afetam a gestão da TI. Em seguida, são analisados os mecanismos de governança de TI adotados e sua relação com o desempenho percebido da gestão da TI.

Com relação à adoção dos mecanismos de governança de TI (Tabela 3), identificou-se que o mecanismo mais utilizado pelas empresas investigadas é o Planejamento Estratégico de TI (PETI) ( $n=75)$. Com a maior diversificação e utilização das aplicações de TI no meio empresarial, muitas empresas têm enxergado o PETI como um mecanismo fundamental para o bom gerenciamento dos recursos de TI (BRODBECK, 2001). Desde o final da década de 1970, uma série de metodologias e técnicas voltadas para a elaboração do PETI vem sendo utilizada pelas organizações, o que caracteriza ser um mecanismo bastante maduro e conhecido entre as empresas. 
Tabela 3: Mecanismos de governança de TI mais utilizados e seu impacto percebido na gestão da TI

\begin{tabular}{|c|c|c|c|}
\hline Mecanismos & $\mathbf{n}$ & Média & Desvio-Padrão \\
\hline Planejamento Estratégico de TI & 75 & 4,03 & ,885 \\
\hline Análise viabilidade de projetos de TI & 72 & 3,68 & ,709 \\
\hline Comitê de projetos de TI & 71 & 4,00 &, 756 \\
\hline Práticas formais de Comunicação & 69 & 3,62 & 893 \\
\hline Participação do CIO na estratégia & 67 & 3,99 & 961 \\
\hline Avaliação pós-implementação & 66 & 3,82 & 927 \\
\hline SLA/SLM & 66 & 3,45 & 880 \\
\hline Compliance & 61 & 4,02 & ,904 \\
\hline Comitês de TI & 59 & 3,88 & 1,001 \\
\hline Práticas para incentivar a gestão da TI & 55 & 3,65 & 821 \\
\hline PMI & 53 & 3,64 & ,787 \\
\hline ITIL & 50 & 3,56 & ,993 \\
\hline Práticas formais de Aprendizagem & 49 & 3,22 & 896 \\
\hline COBIT & 46 & 3,67 & 896 \\
\hline BS7799 & 40 & 3,63 & 1,102 \\
\hline BSC de TI & 36 & 3,89 & ,919 \\
\hline Escritório de Projetos & 36 & 3,78 & 959 \\
\hline ISO9000 & 34 & 3,56 & 1,160 \\
\hline BPM & 31 & 3,32 & ,909 \\
\hline SOA & 28 & 3,32 & ,905 \\
\hline CMM & 23 & 3,17 & 1,072 \\
\hline COSO & 21 & 3,29 & 1,146 \\
\hline 6 sigma & 16 & 3,06 & ,929 \\
\hline
\end{tabular}

Fonte: Elaborada pelos autores

O segundo mecanismo mais utilizado pelas empresas analisadas é a análise de viabilidade de projetos de TI $(n=72)$. Esse mecanismo permite que a organização analise os benefícios e os custos estimados em cada projeto de TI, verificando antecipadamente se o investimento a ser realizado parece valer a pena. Diferentemente das abordagens tradicionais (voltadas exclusivamente para as questões financeiras), as abordagens mais atuais têm buscado combinar os aspectos tangíveis e intangíveis da TI, além de considerar os custos envolvidos e o tempo de implementação.

O terceiro mecanismo mais comum entre as empresas analisadas é o uso de comitês para projetos específicos de TI $(n=71)$. Algumas empresas têm criado estruturas temporárias para discutir o andamento e os interesses de projetos tecnológicos de grande porte, como a implementação de sistemas ERP, e-commerce ou Supply Chain Management. O comitê é 
formado por executivos de TI e de outras áreas de negócio, responsáveis por gerenciarem e acompanharem o andamento do projeto. Assim que o projeto é finalizado, o comitê é extinto. Além desses mecanismos, as práticas formais de comunicação $(n=69)$, a participação do CIO na formulação da estratégia ( $n=67$ ), o uso de métodos de avaliação pósimplementação $(\mathrm{n}=66)$ e os SLAS/SLMs $(\mathrm{n}=66)$ apareceram em um elevado número de empresas analisadas, sugerindo que os executivos, em geral, têm buscado mais informações e conhecimento sobre a função da TI na organização.

Já quanto à percepção dos executivos em relação ao impacto proporcionado pelos mecanismos de governança de TI na gestão da TI (Tabela 3), identificou-se que o planejamento estratégico de TI $(4,03)$, as práticas de compliance (4,02), o comitê de projetos específicos de TI $(4,00)$, a participação do CIO na estratégia da empresa $(3,99)$ e os comitês de TI $(3,88)$ formam o grupo dos mecanismos com maior impacto (percebido) na gestão da TI. Além de ser o mecanismo mais utilizado entre as empresas analisadas, o planejamento estratégico de TI aparece como uma importante ferramenta de gestão da TI, especialmente porque o planejamento busca formalmente definir meios que alinhem a TI aos objetivos de negócios, responsabilizando-se pelo gerenciamento dos recursos tecnológicos, pelo desenvolvimento de políticas e arquiteturas de tecnologia, e pela utilização da TI para obtenção de vantagem competitiva (CHAN et al., 2006).

Além do PETI, o uso de práticas de compliance também foi apontado como um mecanismo de forte impacto sobre a gestão da TI. Pressionadas por diferentes órgãos reguladores, muitas organizações têm se preocupado em garantir a conformidade dos seus processos internos, visto que podem ser auditadas e cobradas por esses órgãos a qualquer momento. Mesmo que não seja responsabilidade exclusiva da área de TI, muitos dos pontos a serem auditados estão relacionados ou são dependentes da TI, como o acesso e a segurança às informações, e a integridade dos sistemas (como o uso de planilhas eletrônicas que ficam sendo manipuladas fora dos sistemas da organização). Com o cumprimento dessas exigências, vários benefícios acabaram atingindo a área de TI, como: a redução do risco de fraudes, a revisão de procedimentos, o desenvolvimento de práticas mais eficientes e uma melhor distribuição de responsabilidades - aspectos anteriormente considerados pela alta administração como superficiais. Em estudo realizado na Bélgica, De Haes e Van Grembergen (2009) identificaram a necessidade de cumprir os 
requisitos da Lei Sarbanes-Oxley como um dos principais motivadores para que os bancos daquele país adotassem a governança de TI.

Já os mecanismos relacionados à estrutura que foram apontados como de grande impacto na gestão da TI, destacam-se o uso de comitês e a participação do CIO na elaboração das estratégias corporativas. Muitas organizações têm utilizado diferentes comitês para auxiliar seus governantes a cumprirem seu dever da melhor forma possível. Dada a importância da TI para os negócios corporativos, tem-se tornado cada vez mais frequente o uso de comitês ligados à TI. Formados por gestores de diferentes áreas, esses comitês têm sido criados com o objetivo de aumentar o comprometimento da organização e a precisão nas decisões ligadas à TI. Embora possa se encontrar uma diversidade deles (Comitê Diretivo de TI, Comitê de Estratégia de TI, Comitê de Governança de TI,...), o mais importante é que os conceitos $e$ a funcionalidade de cada um sejam aplicados e adaptados ao ambiente de cada organização (ITGI, 2003).

Com relação à participação da área de TI - representada pelo $\mathrm{CIO}$ - na definição das estratégias e objetivos corporativos, tem-se percebido que boa parte dos altos executivos não é particularmente versada nos aspectos ligados à tecnologia, o que acaba dificultando a tomada de qualquer decisão ligada à TI. Quando se abre espaço para que a área tecnológica da empresa exponha suas ideias, ou possa influenciar a estratégia e aconselhar os demais executivos quanto a escolhas que venham aperfeiçoar os negócios com a ajuda da TI, verifica-se uma grande oportunidade de ganho para a organização. A forma com que a área de TI tem se juntado à alta administração tem sido através da presença do CIO no Conselho de Administração ou em reuniões com as demais áreas corporativas, o que acaba deixando a organização bem informada sobre os assuntos e as decisões ligadas à TI, além de permitir conhecimento atualizado sobre modelos de negócios, técnicas de gerenciamento, tecnologias, e potenciais riscos e benefícios associados a cada uma das decisões tomadas (DUFFY, 2002).

Com o intuito de analisar os possíveis relacionamentos entre os diferentes mecanismos de governança de TI e o desempenho da gestão de TI na organização (avaliado pelas seis áreas-foco da governança de TI), utilizou-se o coeficiente de correlação de Pearson (Tabela 4). Esse mesmo coeficiente já foi utilizado em outros trabalhos da área de Sistema de Informação (SI), buscando observar as relações existentes entre diferentes variáveis e a tecnologia de informação (KRAEMER; DEDRICK, 1994; CHOE, 2005; YIU, GRANT; EDGAR, 2007). 
Tabela 4: Coeficientes da correlação de Pearson entre os mecanismos de governança de TI e as áreas da governança de TI

\begin{tabular}{|c|c|c|c|c|c|c|}
\hline Mecanismos & MP & VA & RIS & REC & $\mathbf{A E}$ & AC \\
\hline \multicolumn{7}{|l|}{ Estrutura } \\
\hline Comitê de Projetos de TI $(\mathrm{n}=73)$ &, 230 &, $264(*)$ & $279(*)$ & $242(*)$ &, $306(* *)$ &, 213 \\
\hline Participação do CIO $(\mathrm{n}=70)$ & ,297(*) &, $415(* *)$ & ,298(*) & $309(*)$ &, $435(* *)$ & 234 \\
\hline Comitês de TI $(\mathrm{n}=61)$ & $261(*)$ &, $326\left(^{*}\right)$ & $285\left(^{*}\right)$ &, $341(* *)$ &, $331(*)$ & 180 \\
\hline $\mathrm{PMO}(\mathrm{n}=36)$ & $493(* *)$ &, $380(*)$ & 304 & $373(*)$ & $352(*)$ &, $429(* *)$ \\
\hline \multicolumn{7}{|l|}{ Processo } \\
\hline Planejamento Estratégico de TI $(\mathrm{n}=78)$ &, 204 &, 191 & 166 & $344(* *)$ &, $530(* *)$ &, $360(* *)$ \\
\hline Análise de projetos de TI $(\mathrm{n}=75)$ & $421(* *)$ & 173 & 223 & 186 & $415(* *)$ & $462(* *)$ \\
\hline SLA/SLM $(n=69)$ & $315(* *)$ & $273(*)$ & ,302(*) & 138 & $430(* *)$ &, $373(* *)$ \\
\hline Avaliação pós-implementação $(\mathrm{n}=68)$ &, $299(*)$ &, 226 &, 177 & 102 &, 178 & ,261(*) \\
\hline Compliance $(\mathrm{n}=62)$ &, 156 &, 166 & $280\left(^{*}\right)$ & 243 &, $278(*)$ & $263(*)$ \\
\hline PMI $(\mathrm{n}=56)$ &, $322(*)$ & 238 & 136 & 233 &, $367(* *)$ &, 253 \\
\hline $\operatorname{ITIL}(\mathrm{n}=53)$ &, 088 &, 132 & 211 & 271 & 186 &, 072 \\
\hline $\operatorname{COBIT}(\mathrm{n}=48)$ &, 111 &, 148 & 272 & 245 &, 074 &,- 024 \\
\hline BS7799 $(n=41)$ & 245 & 253 &, $330(*)$ & 123 &, $338(*)$ & 179 \\
\hline $\mathrm{BSC}(\mathrm{n}=38)$ & ,432(**) &, $401(*)$ &, $351(*)$ & 207 & ,423(*) & ,404(*) \\
\hline ISO9000 $(n=36)$ &, 159 & 257 & 136 & 260 & $495(* *)$ & $397(*)$ \\
\hline $\mathrm{BPM}(\mathrm{n}=32)$ & 197 &, 127 &, 046 & 019 &, 326 &, 011 \\
\hline $\mathrm{SOA}(\mathrm{n}=28)$ &, 043 &, 049 & 219 &,- 072 &,- 128 &,- 093 \\
\hline $\mathrm{CMM}(\mathrm{n}=23)$ &, 142 &, 174 &, 188 & $423(*)$ &, 341 & 403 \\
\hline $\operatorname{COSO}(n=22)$ &, 070 &,- 032 & 109 & 113 &, 306 &,- 179 \\
\hline 6 Sigma $(n=17)$ &,- 254 &,- 260 &,- 346 & 260 & 231 &,- 023 \\
\hline $\mathrm{BS} 15000(\mathrm{n}=15)$ & 331 & 209 & 252 &,- 268 &,- 030 &,- 161 \\
\hline \multicolumn{7}{|l|}{ Relacionamento } \\
\hline Práticas de Comunicação $(\mathrm{n}=72)$ &, $363(* *)$ &, $347(* *)$ & 190 & $251(*)$ & $421(* *)$ & ,443(**) \\
\hline Práticas de compartilhamento $(\mathrm{n}=57)$ & ,297(*) &, $378(* *)$ &, 102 &, $314(*)$ &, $328(*)$ & 258 \\
\hline Práticas de Aprendizagem Org. $(\mathrm{n}=51)$ & $324(*)$ &, $340(*)$ & $334(*)$ & 238 & 280 & 206 \\
\hline
\end{tabular}

* Correlação significativa ao nível de 0,05

** Correlação significativa ao nível de 0,01

Fonte: Elaborada pelos autores

Pôde-se constatar que dos 24 mecanismos de governança de TI avaliados no estudo, 14 apresentaram correlação positiva significativa (ao nível de $5 \%$ ) com o construto alinhamento estratégico, sendo o PETI $(r=0,530)$, a ISO9000 ( $r=0,495)$, a participação do CIO na definição das estratégias corporativas $(r=0,435)$, os acordos de nível de serviço (SLA/SLM) $(r=0,430)$, as práticas formais de comunicação $(r=0,421)$, a análise de viabilidade de projetos de TI $(r=0,415)$, o PMI $(r=$ $0,367)$ e o comitê de projetos de TI $(r=0,306)$ os mecanismos mais fortemente correlacionados $(p<0,01)$ a esse construto e que, portanto, auxiliariam a organização de forma mais expressiva na tentativa de alinhar as estratégias de TI às estratégias corporativas. 
A literatura de SI tem dado muita atenção às metodologias de desenvolvimento para condução do planejamento estratégico (SEGARS; GROVER, 1998), especialmente porque esses métodos são projetados para auxiliar os planejadores de SI no alinhamento de suas estratégias com as da organização. Quando a organização inclui no planejamento da área de TI o que deve ser adquirido ou desenvolvido - em termos tecnológicos - para potencializar as necessidades competitivas da organização, ela acaba facilitando esse alinhamento (BOWMAN et al., 1983). Brodbeck (2001) constatou, entre um grupo de empresas, que as elas utilizavam-se do planejamento estratégico de TI como forma de promover o alinhamento estratégico entre os negócios e a TI, em maior ou menor intensidade, mesmo que em algumas etapas do processo de planejamento isso fosse realizado de forma empírica. A mesma autora identificou uma série de elementos que deveriam estar presentes no PETI de modo a promover o alinhamento estratégico (como o horizonte de planejamento; a transcrição dos objetivos em projetos, programas ou indicadores; o monitoramento dos objetivos organizacionais; o comprometimento dos integrantes da organização, dentre outros).

Chan et al. (2006) constataram que a sofisticação e a compreensão do planejamento estratégico de TI levam a um aumento no compartilhamento do conhecimento, que por sua vez, afeta o alinhamento. Quanto mais sofisticado for o processo de planejamento, maior será a probabilidade de envolvimento de pessoas de diferentes áreas da empresa - tudo isso levando ao alinhamento. Com a área de TI passando a ocupar um lugar cada vez mais estratégico nas organizações, a qualidade dos serviços prestados por ela acaba afetando diretamente a qualidade e a eficiência do trabalho realizado pelas demais áreas. Dessa forma, percebe-se entre as organizações certa consciência na necessidade de adotar boas práticas de gestão que garantam que os investimentos em TI e o aumento gradativo da complexidade dos serviços não comprometam a organização. Nesse contexto, desponta a ISO9000 e as suas variações como uma forma de melhorar os resultados da organização, encorajando a melhoria contínua ou a prevenção de defeitos (CORBETT; MONTES-SANCHO; KIRSCH, 2005).

Além da ISO9000, destaca-se o uso dos acordos de nível de serviço SLAs (do inglês Service Level Agreement) como forma de monitoramento do alinhamento estratégico (SLEDGIANOWSKI; LUFTMAN, 2005). Os SLAs deixaram de ser um checklist apenas técnico para assumirem um papel de suporte à melhoria dos processos de negócios (sendo chamados pelos exe- 
cutivos de a "segunda onda dos SLAs"), ("Vem aí a segunda onda do SLA", Info Corporate, n. 43, abril de 2007). No lugar de uma extensa lista técnica, com até 100 itens a serem checados, a nova onda de SLAs abrange poucos indicadores, de cinco a dez, em média, mas totalmente orientados a negócios. O enunciado muda conforme as características de cada empresa, mas os indicadores são divididos em três grupos: 1) qualidade, determinada pela disponibilidade, número de falhas, conformidade ou satisfação dos usuários; 2) tempo de resposta, medido da solicitação ao completo atendimento; $e$ 3) eficiência, medida pela unidade de esforço, utilização da equipe ou pelo índice de retrabalho.

Outro construto que apresentou um elevado número (12) de mecanismos de governança de TI apresentando correlação positiva significativa foi o construto medidas de performance. Os mecanismos com maior correlação foram, respectivamente, a presença de um escritório de projetos $(r=$ 0,493), o uso do balanced scorecard (BSC) ( $r=0,432)$, o uso de métodos de análise de viabilidade de projetos de TI $(r=0,421)$, os acordos de nível de serviço $(r=0,315)$ e as práticas de comunicação $(r=$ 0,363). O grande número de projetos de TI que acaba atrasando, superando o orçamento ou não apresentando os resultados previstos, é um problema bastante antigo, mas que continua preocupando bastante os executivos (MAIZLISH; HANDLER, 2005), o que tem feito com que as questões relacionadas ao seu gerenciamento sejam consideradas um assunto extremamente importante (KUMAR, 2002). Tanto o escritório de projetos, como o uso do $\mathrm{BSC}$ e de métodos de análise de viabilidade possuem características bastante comuns (como a utilização de diferentes indicadores quantitativos para estimar, comparar e acompanhar os projetos de TI), permitindo avaliar o andamento e o impacto proporcionado pelos projetos tecnológicos da organização. Segundo Grover, Kearns e Sabherwal (2006-2007), a qualidade do planejamento e da implementação dos projetos de TI media também o relacionamento entre o alinhamento estratégico de TI e de negócios e o efeito da TI nos negócios.

Já os mecanismos que apresentaram o maior número de correlações significativas com os construtos avaliados foram: o comitê de projetos de TI, as práticas formais de comunicação, a participação do CIO na formulação da estratégia da organização, os acordos de nível de serviço (SLA/SLM), a presença de comitês de TI e do escritório de projetos, e o uso do balanced scorecard. Chama a atenção o fato de 
todos os mecanismos de governança relativos à estrutura - listados no questionário - terem apresentado correlação positiva significativa com os construtos avaliados, destacando o impacto que a presença de estruturas físicas formais têm no desempenho da gestão da TI (seja decidindo quando um investimento deve ou não ser realizado, ou acompanhando os projetos em andamento, ou ainda garantindo um maior envolvimento entre a área de negócios da organização e a área de TI) (SAMBAMURTHY; ZMUD, 1999).

Quanto às práticas formais de comunicação, Johnson e Lederer (2005) apontam a comunicação efetiva entre todas as partes (baseada em relacionamentos construtivos, com uma linguagem comum e o comprometimento compartilhado das políticas e procedimentos de $\mathrm{TI}$ ) como um fator essencial para o sucesso da governança de TI. No mesmo sentido, Sledgianowski e Luftman (2005) falam da comunicação como um dos principais mecanismos de gestão de TI. Os autores defendem que a comunicação deveria ser uma tarefa fundamental, ocorrendo regularmente entre os gerentes e os funcionários. A comunicação entre a área de TI e as demais áreas de negócios pode ser tanto informal quanto utilizando métodos apropriados como e-mail, videoconferência e comunicação face a face. Os mesmos autores sugerem também o uso dos SLAs como um importante meio de monitorar o alinhamento estratégico, seja através de avaliações periódicas formais ou revisões dos acordos preestabelecidos, envolvendo representantes da área de TI e de negócios, formalizando as mudanças baseadas nos resultados das avaliações e justificando a sua forte correlação com os construtos de governança de TI já avaliados.

As práticas formais de aprendizagem organizacional - como o uso de bancos de projetos, rotação de cargos e funções, treinamento interfuncional entre TI e outras áreas - e as práticas que incentivam uma gestão compartilhada de $\mathbf{T I}$ - como a participação de diferentes gestores nos projetos de TI ou, ainda, a área de TI ou de negócios dando sugestões de projetos a serem realizados ou melhorados - merecem um breve destaque. Embora tenham apresentado correlações significativas mais fracas que outros mecanismos, pôde-se perceber que, de modo geral, a sua utilização reflete positivamente sobre a gestão da $\mathrm{TI}$, especialmente quanto aos aspectos ligados ao cumprimento de prazos, recursos despendidos e benefícios obtidos a partir da implementação de diferentes projetos de TI.

Outro fato bastante interessante foi verificar que os modelos ITIL $e$ COBIT não apresentaram correlação significativa com nenhum dos 
construtos avaliados. Tanto um quanto o outro se constituem de um conjunto de componentes - compostos por diferentes mecanismos - que nessa configuração macro não apresentou correlação significativa. Entretanto, quando alguns dos seus componentes são analisados individualmente - como o caso do PETI, do uso de comitês, normas de segurança, acordos de nível de serviço, entre outros mecanismos presentes nos dois modelos - acabam se mostrando fortemente correlacionados com diversos construtos. Tanto o COBIT quanto o ITIL atuam como um guia de referência na gestão da TI, não exigindo, entretanto, que todos os seus processos e objetivos de controle sejam adotados.

Ainda sobre os mecanismos, verificou-se uma correlação positiva entre o CMM e o construto gerenciamento de recursos. O CMM pode ser compreendido como um conjunto de "melhores práticas" para diagnóstico e avaliação de maturidade do desenvolvimento de software em uma organização. Ele fornece orientação sobre como controlar esse processo de desenvolvimento e como evoluir para uma cultura de excelência na gestão de software (não apenas quanto ao desenvolvimento, mas também na aquisição de sistemas, na qualidade dos recursos humanos, etc.). Assim, espera-se que os recursos de TI da organização funcionem de forma adequada e não comprometam o andamento das suas atividades operacionais, justificando a exigência dessa certificação na hora de desenvolver ou adquirir um sistema qualquer.

Percebeu-se através dos coeficientes de Pearson que vários mecanismos de governança de TI apresentaram correlação positiva significativa com os construtos avaliados, sugerindo uma associação entre esses mecanismos e o desempenho da gestão de TI na organização. Essas correlações podem indicar aos executivos quais mecanismos devem ser prioritariamente adotados, evidenciando como eles se correlacionam com o desempenho da gestão da TI. Bowen, Cheung e Rohde (2007) avaliaram diferentes fatores que influenciavam os processos, as estruturas e os resultados da governança de TI, concluindo que o desempenho da governança estava associado à compreensão dos objetivos de TI e de negócios, do envolvimento ativo dos comitês de TI, de um número balanceado de representantes de TI e de negócios nas decisões referentes à TI, e à presença de políticas e estratégias de TI compreensíveis e bem comunicadas. Os autores identificaram também que o desempenho da governança de TI aumenta o sucesso dos projetos de TI e a entrega de valor de negócios. 
A correspondência entre alguns resultados encontrados nesta investigação e alguns dos resultados encontrados por Bowen et al. (2007), embora realizados em contextos distintos e utilizando diferentes metodologias, instigam os autores a propor um modelo relacionando a governança de TI e seu impacto na gestão de TI, com destaque para os mecanismos mais relevantes encontrados nesta pesquisa. A Figura 2 ilustra a especulação.

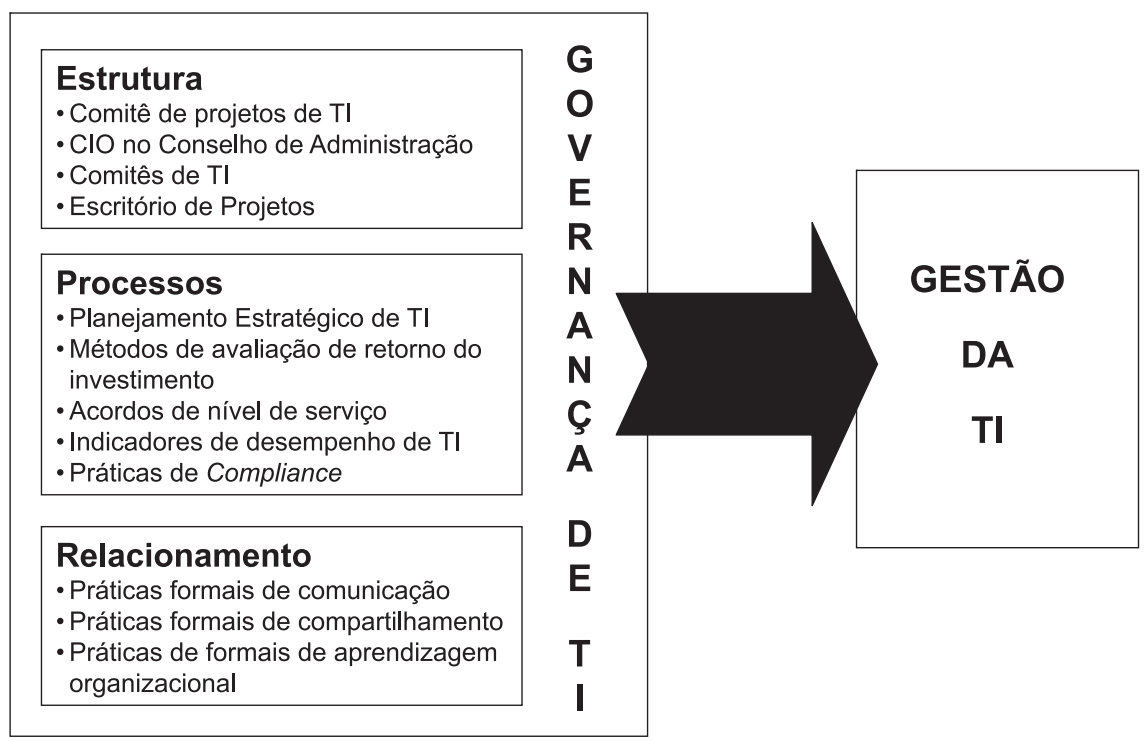

Figura 2: Modelo de governança de TI e seu impacto na gestão da TI Fonte: Elaborada pelos autores

\section{Considerações Finais}

O presente estudo buscou avaliar, sob a percepção dos executivos, o impacto da implementação de diferentes mecanismos de governança de TI no desempenho da gestão da TI. Dos 24 mecanismos analisados, o PETI, a análise de viabilidade de projetos de TI e o uso de comitês para projetos específicos de TI aparecem como os mecanismos mais utilizados pelas empresas analisadas. Os mecanismos que mais afetam o desempenho (percebido) da gestão da TI são o PETI, o comitê de projetos específicos de TI, a participação do $\mathrm{CIO}$ na formulação da estratégia da empresa, as práticas de compliance e os comitês de TI. 
Os resultados da investigação sugerem que quanto mais efetivos forem os mecanismos de governança de TI, melhor será o desempenho da gestão de TI na organização. Os mecanismos são ferramentas de gestão de TI, muitas delas consolidadas no mercado e utilizadas por diferentes empresas com o intuito de potencializar um ou outro aspecto da gestão dos seus recursos de TI. Os mecanismos mais fortemente associados com as áreas foco da governança de TI são: (1) o comitê de projetos de TI; (2) as práticas formais de comunicação; (3) a participação do $\mathrm{CIO}$ na formulação da estratégia da organização; (4) os acordos de nível de serviço (SLA/SLM); (5) a presença de comitês de TI; (6) o uso do escritório de projetos; e (7) o uso do balanced scorecard. Tais resultados corroboram os também encontrados por Bowen et al. (2007). Em um estudo de caso realizado em uma grande empresa (mais de 3.000 funcionários, cinco unidades de negócios, com investimentos em TI na ordem de 200 milhões de dólares) com atuação na Austrália e Nova Zelândia, os autores concluem que níveis mais altos de efetividade da governança de TI estão associados com níveis superiores de compartilhamento do entendimento acerca dos objetivos de negócios e de TI entre os executivos, um papel mais ativo dos comitês de TI, e um melhor balanceamento na representação entre executivos de negócios e de TI nos comitês de TI, além de possuir políticas e estratégias de TI bem comunicadas e compreendidas.

Pôde-se constatar também que todos os mecanismos relativos à estrutura - listados no questionário (como os comitês de projetos de TI, os comitês de TI, os escritórios de projetos e a participação da área de TI na formulação da estratégia corporativa) - estão associados com as áreas foco da governança de TI, revelando a importância da presença de estruturas formais no desempenho da gestão da TI (seja auxiliando na decisão de se fazer ou não um investimento em TI, acompanhando os projetos em andamento, ou ainda garantindo um maior envolvimento entre a área de negócios da organização e a área de TI).

Com relação às contribuições desta pesquisa, destacam-se: a elaboração e validação de um instrumento capaz de avaliar o desempenho da gestão da TI pelas organizações, além da identificação dos mecanismos de governança de TI mais comuns entre as organizações estudadas e dos que possuem maior impacto sobre a gestão da TI e seus diferentes aspectos. Essas implicações podem servir como um guia prático para os executivos de TI e de negócios que estejam implementando ou desejando implementar a governança de TI ou alguns de seus mecanismos. Como contribuição teóri- 
ca, propõe-se um modelo de governança de TI e seu impacto na gestão da TI (Figura 2).

Como limitações do estudo, destaca-se que a seleção dos construtos teóricos propostos para avaliar a gestão da TI não garante que todos os aspectos da governança de TI tenham sido incluídos no instrumento de pesquisa. Entretanto, a proposição dos seis construtos analisados se deu após a realização de uma extensa revisão de literatura, selecionando-se aqueles mais frequentemente citados, o que incorpora maior credibilidade ao instrumento proposto. Outra potencial limitação corresponde à natureza e tamanho da amostra utilizada nas análises. A baixa taxa de retorno dos questionários referentes às empresas listadas na BOVESPA (cerca de 20\%) e o acréscimo dos questionários utilizados no estudo-piloto à amostra final sugerem cuidados na interpretação dos resultados obtidos, descartando a sua possibilidade de generalização, e restringindo suas conclusões apenas as 81 empresas analisadas. Cabe salientar que em 79 das 81 empresas que participaram da pesquisa survey, um único informante respondeu à pesquisa, o que embora seja bastante comum entre os estudos realizados na área de SI, pode não ser o melhor método para a obtenção de dados mais precisos sobre a organização (PINSONNEAULT; KRAEMER, 1993).

\section{The Impact of Adopting IT Governance Mechanisms on IT Management Performance: an Analysis regarding the executive's perception}

\section{Abstract}

Recently, there has been a great deal of interest on the part of many executives the concept of IT governance in order to justify IT investments. However some studies have shown that companies which have good IT governance models generate higher returns on their IT investments than their competitors, less research can be found on how organizations are effectively implementing IT governance in day-to-day practice. Thus, we evaluated the perceived impact of different IT governance mechanisms on IT management performance. The study was done with 83 IT managers, indicating the most adopted IT governance mechanisms as well as their impact on IT management. Results indicated a great number of IT governance 
mechanisms correlating positively with different IT governance domains, highlighting IT Strategic Planning, IT projects Committee, CIO on board of directors, compliance practices and IT committees as the most important mechanisms, suggesting the more effective they were, the better IT management will be.

Key words: IT Governance. IT Management. Mechanisms. Impact. Performance.

\section{Referências}

ALBERTIN, R. Governança de Tecnologia de Informação: estrutura e práticas. 2008. Tese (Doutorado em Administração de Empresas) - Escola de Administração de Empresas de São Paulo da Fundação Getúlio Vargas, São Paulo, 2008.

BORGMAN, H.; HEIER, H. Ricochet of the magic bullet: revisiting the role of change management initiatives in IT Governance. Proceedings of the 43th Hawaii International Conference on System Sciences, Hawaii, 2010.

BOWEN, P. et al. Enhancing IT governance practices: a model and case study of an organization's efforts. International Journal of Accounting Information Systems, v. 8, p. 191-221, 2007.

BOWMAN, A. et al. Three stage model of MIS planning. Information \& Management, v. 6, n. 3, p. 11-25, 1983.

BRETERNITZ, V.; NAVARRO NETO, F.; NAVARRO, A. Gerenciamento de segurança segundo ITIL: um estudo de caso em um organização industrial de grande porte. Revista Eletrônica de Sistemas de Informação, v. 8, n. 2, artigo 4, p. 1-15, 2009.

BRODBECK, A. Alinhamento estratégico entre os planos de negócio e de Tecnologia de Informação: um Modelo Operacional para a Implementação. 2001. Tese (Doutorado em Administração de Empresas) Programa de Pós Graduação em Administração, Escola de Administração, Universidade Federal do Rio Grande do Sul, 2001.

BROWN, A.; GRANT, G. Framing the frameworks: a review of IT governance research. Communications of the Association for Information Systems, v. 15, p. 696-712, 2005. 
BROWN, W. IT governance, architectural competency, and the Vasa. Information Management \& Computer Security, v. 14, n. 2, p. 140-154, 2006.

CHAN, Y. et al. Antecedents and outcomes of strategic IS alignment: an empirical investigation, IEEE Transactions on Engineering Management, v. 53 , n. 1 , p. $27-47,2006$.

$\mathrm{CHOE}$, J. The relationships among management accounting information, organizational learning and production performance. Journal of Strategic Information Systems, v. 13, n. 1, p. 61-85, 2005.

CHURCHILL, G. A. A paradigm for developing better measures of marketing constructs. Journal of Marketing Research, v. 16, p. 64-73, 1979.

CORBETT, C.; MONTES-SANCHO, M.; KIRSCH, D. The financial impact of ISO 9000 certification in the United States: an empirical analysis.

Management Science, v. 51, n. 7, 2005.

DE HAES, S.; VAN GREMBERGER, W. An exploratory study into IT Governance implementations and its impact on business/IT alignment.

Information Systems Management, 26, p. 123-137, 2009.

DETLOR, B. Information management. International Journal of Information Management, 30, p. 103-108, 2010.

DUFFY, J. IT Governance and business value part 2: who's responsible for what? IDC document, n. 27807, 2002.

FARBEY, B.; LAND, F.; TARGETT, D. Evaluating investments in information technology. Journal of Information Technology, v. 7, n. 2, p. 109-122, 1992

GELLINGS, C. Outsourcings relationships: the contract as IT governance tool. Proceedings of the 40th Hawaii International Conference on System Sciences, Hawaii, 2007.

GROVER, V.; KEARNS, S.; SABHERWAL, R. Strategic alignment between business and Information Technology: a knowledge-based view of behaviors, outcome, and consequences. Journal of Management Information Systems, v. 23, n. 3, p. 129-162, 2006-2007.

GWILLIM, D.; DOVEY, K.; WIEDER, B. The politics of post-implementation reviews. Information Systems Journal, v. 15, p. 307-319, 2005.

HARDY, G. Using IT governance and COBIT to deliver value with IT and respond to legal, regulatory and compliance challenges. Information Security technical report, p. 55-61, March 2006. 
ISACA. Top Business/Technology issues: survey results, 2008. Disponível em: <http://www.itgi.org/

Template. $\mathrm{cfm}$ ?Section $=$ Home $\&$ CONTENTID $=43978 \&$ TEMPLATE $=/$

ContentManagement/ContentDisplay.cfm > . Acesso em: 28 maio 2010.

ITGI. Board briefing on IT governance. Rolling Meadows/EUA: IT Governance Institute, 2. ed. 2003.

ITGI. Cobit 4.0: Control objectives management guidelines maturity models. Rolling Meadows/EUA: IT Governance Institute, 2005.

JOHNSON, A.; LEDERER, A. The effect of communication frequency and channel richness on the convergence between chief executive and chief information officers. Journal of Management Information Systems, v. 22, n. 2, p. 227-252, 2005.

KOUFTEROS, X. Testing a model of pull production: a paradigm for manufacturing research using structural equation modeling. Journal of Operations Management, v. 17, n. 4, p. 467-488, 1999.

KRAEMER, K.; DEDRICK, J. Payoffs from Investment in Information Technology: Lessons from the Asia-Pacific Region. World Development, v. 22, n. 4, p. 1921-1931, 1994.

KUMAR, R. Managing risks in IT projects: an options perspective. Information \& Management, v. 40, n. 1, p. 63-74, 2002.

LUNARDI, G.; DOLCI, P. Governança de TI e seus mecanismos: uma análise da sua disseminação entre as empresas brasileiras. In: ENCONTRO DE ADMINISTRAÇÃO DA INFORMAÇÃO - ENADI, 2, 2009, Recife. Anais... Curitiba: ANPAD, 2009. 1 CD-ROM.

MAIZLISH, B.; HANDLER, R. IT portfolio management: step by step. New Jersey: John Wiley \& Sons, 2005.

McLANE, G. IT governance and its impact on IT management (a literature review). 2003. M.B Dissertation Project (Masters of Business in Information Technology Management) - University of Technology Sydney, UTS, Sydney, 2003.

MEYER, N. Systemic IS governance: an introduction. Information Systems Management, v. 21, p. 23-34, 2004.

NEVO, S.; WADE, M.R. The formation and value of IT-enabled resources: antecedents and consequences of synergistic relationships. MIS Quarterly, v. 34, n. 1, p. 163-183, 2010. 
PETERSON, R. Integration strategies and tactics for information technology governance. In: VAN GREMBERGEN, W. Strategies for information technology governance, Hershey: Idea group publishing, 2004.

PINSONNEAULT, A.; KRAEMER, K. Survey research methodology in management information systems: an assessment. Journal of Management Information Systems, v. 10, n. 2, p. 75-106, 1993.

SAMBAMURTHY, V.; ZMUD, R. Arrangements for information technology governance: a theory of multiple contingencies. MIS Quarterly, v. 23, n. 2, p. 261-290, 1999.

SEGARS, A.; GROVER, V. Strategic information systems planning success: an investigation of the construct and its measurement. MIS Quarterly, v. 22, n. 2, p. 139-163, 1998.

SLEDGIANOWSKI, D.; LUFTMAN, J. IT-business strategic alignment maturity: a case study. Journal of Cases on Information Technology, v. 7, n. 2, p. 102-120, 2005.

TAROUCO, H.; GRAEML, A. Governança de Tecnologia da Informação: um panorama da adoção de modelos de melhores práticas por empresas brasileiras usuárias de TI. In: ENCONTRO DE ADMINISTRAÇÃO DA INFORMAÇÃO ENADI, 2., 2009, Recife. Anais... Curitiba: ANPAD, 2009. 1 CD-ROM.

VAN GREMBERGEN, W.; DE HAES, S.; GULDENTOPS, E. Structures, processes and relational mechanisms for IT governance. In: VAN GREMBERGEN, W. Strategies for information technology governance, Hershey: Idea group publishing, 2004.

VERHOEF, C. Quantifying the effects of IT-governance rules. Science of Computer Programming, v. 67, n. 2-3, p. 247-277, 2007.

WEBB, P.; POLLARD, C.; RIDLEY, G. Attempting to define IT governance: wisdom or folly? Proceedings of the $3^{\text {th }}$ Hawaii International Conference on System Sciences, Hawaii, 2006.

WEILL, P.; ROSS, J. A matrix approach to designing IT governance. Sloan Management Review, v. 46, n. 2, p. 26-34, 2005.

WILLSON, P.; POLLARD, C. Exploring IT governance in theory and practice in a large multi-national organisation in Australia. Information Systems Management, 26, p. 98-109, 2009.

YIU, C.; GRANT, K.; EDGAR, D. Factors affecting the adoption of Internet Banking in Hong Kong - implications for the banking sector. International Journal of Information Management, v. 27, n. 5, p. 336-351, 2007. 